\title{
Development of a 5-fluorouracil-loaded PLGA microsphere delivery system by a solid-in-oil-in-hydrophilic oil (S/O/hO) novel method for the treatment of tumors
}

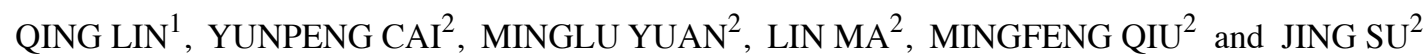 \\ ${ }^{1}$ Shanghai Tenth People's Hospital, Shanghai 200072; ${ }^{2}$ School of Pharmacy, \\ Shanghai JiaoTong University, Shanghai 200240, P.R. China
}

Received April 30, 2014; Accepted July 29, 2014

DOI: $10.3892 / o r .2014 .3480$

\begin{abstract}
Tumor treatment requires a long-term regimen of chemotherapy, and both surgical tumor resection and radiation therapy are also used. The present study aimed to develop a novel method for 5-fluorouracil (5-FU)-loaded microspheres which enhance the therapeutic effects of chemotherapy, the quality of life of patients and reduce chemotherapy systemic side-effects. The preparation of a 5-FU microsphere delivery system by a solid-in-oil-in-hydrophilic oil $(\mathrm{S} / \mathrm{O} / \mathrm{hO})$ novel method was carried out and then in vitro and in vivo evaluation of the 5-FU-microsphere delivery system was conducted. The 5-FU microsphere delivery system prepared had sustainedrelease function and achieved local treatment efficacy for tumors. The encapsulation efficiency of the 5-FU microsphere delivery system was $>90 \%$ [better than the fabrication method using water-in-oil-in-water $(\mathrm{W} / \mathrm{O} / \mathrm{W})]$. The drug release profile from the 5-FU-loaded sustained-release microsphere delivery system matched the pseudo zero-order equation for 30 days in vitro. The plasma concentration of 5-FU was higher than the water solution by subcutaneous injection. The tumor growth rate of rabbits using the 5-FU microsphere delivery system was much lower than the rate in rabbit using a subcutaneous injection of 5-FU water solution. The 5-FU-loaded sustainedrelease microspheres using the novel method $(\mathrm{S} / \mathrm{O} / \mathrm{hO})$ is a potential and effective method with which to inhibit tumor growth.
\end{abstract}

\section{Introduction}

5-Fluorouracil (5-FU) is one of the classical drugs for tumor chemotherapy $(1,2)$. It has been used for colorectal tumors (3-6),

Correspondence to: Professor Mingfeng Qiu or Dr Jing Su, School of Pharmacy, Shanghai JiaoTong University, 800 Dongchuan Road, Shanghai 200240, P.R. China

E-mail:mfqiu@sjtu.edu.cn

E-mail: jingsu@sjtu.edu.cn

Key words: microspheres, sustained-release, delivery system, cancer pancreatic cancer $(5,6)$, actinic keratosis $(7,8)$ and breast tumors (9-12). As a systemic injection of 5-FU often leads to systemic toxic side-effects, tumor in situ sustained-release microspheres or a hydrogel delivery system will certainly improve the antitumor effects of the drug $(13,14)$. Different delivery systems for 5-FU have been developed, including microsphere delivery systems (15), nanospheres (16), 5-FU microspheres for brain tumor therapy (17), 5-FU microspheres for malignant glioma therapy (18), 5-FU hydrogels for drug administration in vivo (19), matrix microspheres containing a 5-FU coat using Eudragit S100 (20), and nanoscale-particles for topical delivery (21), to name just a few (22-29). The specific delivery system can reduce the systemic toxicity and prevent anticancer drug degradation (30-33). However, different methods have different weaknesses such as low encapsulation efficiency, burst release or insufficient duration for drug release.

We previously developed various preparation methods for biological molecular protein drug delivery such as the waterin-oil-in-hydrophilic oil-in-water $(\mathrm{W} / \mathrm{O} / \mathrm{hO} / \mathrm{W})$ method (34), solid-in-oil-in-oil-in-water (S/O/O/W) method (35-37), and solid-in-oil-in-hydrophilic oil-in-ethanol $(\mathrm{S} / \mathrm{O} / \mathrm{hO} / \mathrm{E})$ method $(38,39,40)$. The chemical agents were not microencapsulated in the microspheres using these preparation methods. The present study aimed to develop a 5-FU-loaded sustained-release microsphere system using a novel method solid-in-oil-in-hydrophilic oil $(\mathrm{S} / \mathrm{O} / \mathrm{hO})$ method and to study the characteristics of the microspheres using the novel method in vivo and in vitro.

\section{Materials and methods}

Materials. Ethyl acetate (analytical reagent), dichloromethane (DCM; chemical reagent) and 5-FU were purchased from Sigma-Aldrich. Poly(dl-lactide-co-glycolic acid) (PLGA; $\mathrm{L}: \mathrm{G}=50: 50$, MW $47 \mathrm{kDa}$ ) was purchased from Lakeshore Biomaterials, Inc. (USA).

Animals. Purebred male rabbits, 2-3 months of age, weighing $2.0-2.5 \mathrm{~kg}$ were used for the efficacy study in vivo. Adult male Sprague Dawley (SD) rats $(200.2 \pm 25.1 \mathrm{~g})$ were used for determining the in vivo sustained-release of the 5-FU concentration 
in the blood. SD rats and rabbits were purchased from the Shanghai SLAC Laboratory Animal Co. Ltd. Animals were kept under standardized rodent conditions at $22.2 \pm 1.2^{\circ} \mathrm{C}$.

Preparation of the 5-FU microsphere delivery system by the novel method of $\mathrm{S} / \mathrm{O} / \mathrm{hO}$. The 5-FU microsphere delivery system was placed in dichloromethane solution of PLGA. After stirring for $1 \mathrm{~min}$, the suspension was transferred into the hydrophilic oil phase (hO; containing $73 \% \mathrm{w} / \mathrm{w}$ ethylene glycol, 9\% w/w PVA and 18\% w/w glycerol) to become embryonic microspheres. The embryonic microsphere emulsion was then placed into sodium chloride solution $(10 \%, \mathrm{w} / \mathrm{w})$ and stirred for $2 \mathrm{~h}$. The microspheres were gathered through centrifugation and freeze drying before usage. The control microspheres were prepared using a water-in-oil-in-water (W/O/W) method.

Surface morphology of the 5-FU microsphere delivery system. The size and surface morphology of the 5-FU microsphere delivery system were determined using a Hitachi S-4700 scanning electronic microscope (SEM). These microspheres were coated with gold in a vacuum before being scanned by SEM and the coating procedure was repeated three times.

Encapsulation efficiency of the 5-FU microsphere delivery system. 5-FU microspheres (12 $\mathrm{mg})$ were weighed and suspended in $5 \mathrm{ml} \mathrm{DCM}$, and then the suspension was applied to centrifugation at $12,000 \mathrm{rpm}$ for $6 \mathrm{~min}$ to remove PLGA. The remaining 5-FU microparticles were gathered by washing and centrifugation for an additional three times. Finally, the DCM solvent residues were removed by using evaporation in vacuum drying. The amount of 5-FU in the microsphere delivery system was determined by HPLC. The encapsulation efficiency (EE) was calculated using the formula as below:

$\mathrm{EE}(\%)=\frac{\text { actual amount of drug loaded in microsphere }}{\text { theoretical amount of drug loaded in microsphere }} \times 100$

In vitro release. The release tests were performed at $37^{\circ} \mathrm{C}$. Microspheres (12 mg) weighed were re-suspended in $1 \mathrm{ml}$ PBS ( $\mathrm{pH}$ 7.4) release medium. Then the samples were incubated in a simulating biological fluid incubator at $37^{\circ} \mathrm{C}$ under a shaking rate of $150 \pm 10 \mathrm{rpm}$. The release medium containing the microspheres was centrifuged, and then the supernatant of the samples was completely drawn for each day, and another $1 \mathrm{ml}$ of fresh release medium was then placed. The concentration of the 5-FU drug was measured using HPLC. Average and standard deviation of the data were obtained from three repeats of the same experiment.

In vivo release of the drug concentration in plasma. After the subcutaneous injection of the 5-FU microsphere delivery system and administration of the 5-FU solution, the rats were initially anesthetized using halothane before performing the experiments. Blood $(0.85 \mathrm{ml})$ was gathered from each rat at specific intervals following injection using puncture of the eye vein and collected in heparinized (15 $\mu \mathrm{l}$ containing 75 units) polypropylene tubes. The samples were centrifuged at 12,000 x $\mathrm{g}$ for $14 \mathrm{~min}$ in an Eppendorf $\mathrm{AG}$ (5414 D) centrifuge, and the samples were then collected to gain plasma samples. Proteins in the plasma were removed through precipitation by adding $2 \mathrm{M}$ trichloroacetic acid (6 $\mu \mathrm{l} \mathrm{TCA} / 100 \mu \mathrm{l}$ plasma), and then centrifuged $(12,000 \mathrm{x} \mathrm{g}$, $5 \mathrm{~min}$ ), and finally the plasma was placed at $-20^{\circ} \mathrm{C}$. $5-\mathrm{FU}$ was extracted from this plasma using $6 \mathrm{ml}$ ethyl acetate (41) and $100 \mu \mathrm{l}$ PBS $(0.5 \mathrm{M}, \mathrm{pH} 8.0)$ was placed into $500 \mu \mathrm{l}$ plasma. The samples were then shaken for $7 \mathrm{~min}$ and centrifuged (4,200 x g, $3 \mathrm{~min})$, and the ethyl acetate phase was gathered. The ethyl acetate phase was removed by nitrogen gas at $55^{\circ} \mathrm{C}$, and then the dried samples were re-dissolved with $100 \mu \mathrm{l}$ $\left(0.01 \mathrm{M} \mathrm{pH} 4.0\right.$ of potassium dihydrogen phosphate $\left(\mathrm{KH}_{2} \mathrm{PO}_{4}\right)$, and the 5-FU drug concentration in the plasma was measured by HPLC (Shimadzu LC-10ATVP, Japan). The reverse phase (Waters) chromatographic $\mathrm{C} 18$ column was used. The mobile phase was $0.01 \mathrm{M} \mathrm{pH} 4.0$ of $\mathrm{KH}_{2} \mathrm{PO}_{4}$. The flow speed of the mobile phase was $1 \mathrm{ml} / \mathrm{min}$, and a wavelength of $266 \mathrm{~nm}$ of the detector was used for measurement of the drug concentration. To determine the content of 5-FU, 5-FU standards of $0.1-100 \mu \mathrm{g} / \mathrm{ml}$ in PBS (1 mM, pH 7.4) were used. Drug-free plasma samples pooled with a known content of 5-FU were used for samples containing plasma. In both cases, 5-FU standard profiles were determined using an external standardization method and linear profiles with a correlation coefficient of 0.990 obtained from the area under the UV absorption peak determinations. The retention time of 5-FU drug was $10.5 \pm 0.5 \mathrm{~min}$. From the area under the absorption peak determinations of the plasma 5-FU concentration and retention time, a decreased rate of 5-FU drug concentration in the rat plasma was calculated through the slope of the straight line from the time of maximum 5-FU drug concentration, the absence of drug in rat plasma, and the circulating SD rat blood volume. For calculating the circulating SD rat blood volume, the suggested average value of $64 \mathrm{ml} / \mathrm{kg}$ body weight was calculated (41).

5-FU microsphere delivery system administered for in vivo efficacy investigation. The local antitumor efficacy studies were carried out in rabbits with colon tumors. All rabbit experiments were performed according to protocols approved by the Institutional Animal Care and Use Committee of Shanghai JiaoTong University. Purebred male rabbits, 2-3 months of age, weighing 2.0-2.5 kg were used. After anesthesia, the proximal lateral side of the thigh skin was routinely shaved, disinfected, and the medial side of the thigh muscle of each rabbit was explanted with $1 \mathrm{~mm}^{3}$ fresh tumor tissue blocks. Continuous observation of tumor growth was carried out. Tumors $\sim 2 \mathrm{~cm}$ in diameter were noted in all rabbits two weeks after the transplantation. Eighteen rabbits were separated into three groups, namely: i) no therapy group (blank group); ii) single intravenous 5-FU treatment group; and iii) microsphere administered group. All rabbits after anesthesia underwent CT scanning, three-dimensional outline tumor on CT image processor workstations, and measurement of tumor size.

Statistical analysis of data. The data are expressed as the average \pm standard deviation, and statistically significant differences were determined using one-way ANOVA test. $\mathrm{P}<0.05$ was considered to indicate a statistically significant difference. 

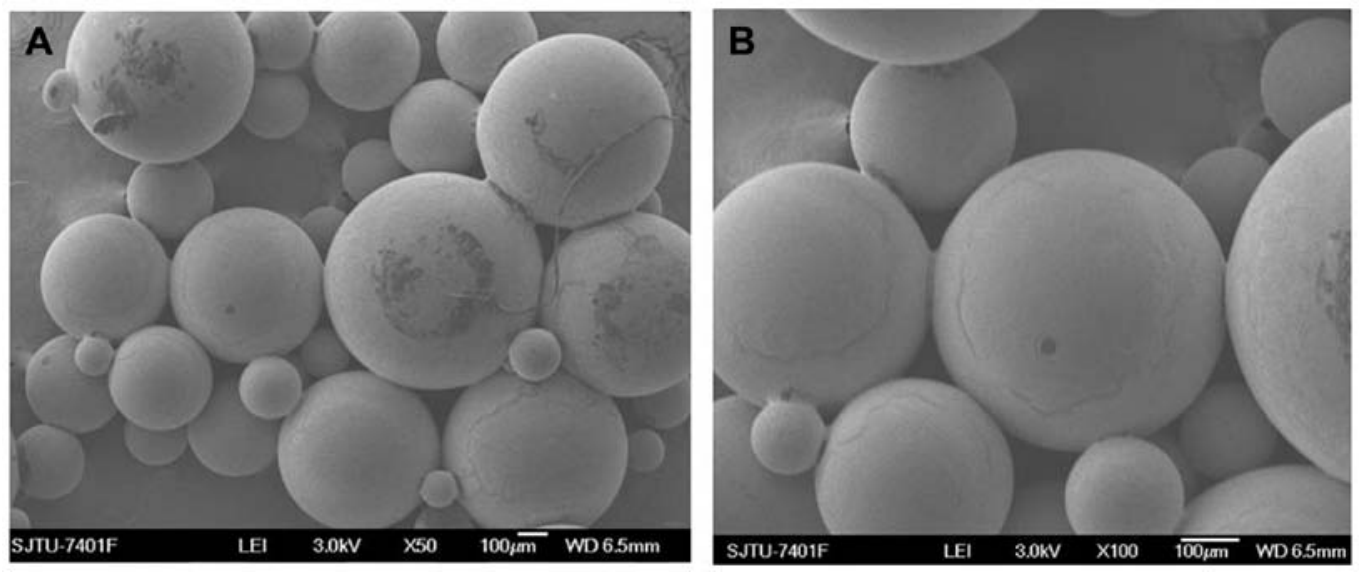

Figure 1. SEM of the 5-FU microsphere delivery system. (A) Blank PLGA microsphere delivery system; (B) 5-FU microsphere delivery system. SEM, scanning electronic microscope; 5-FU, 5-fluorouracil; PLGA, poly(dl-lactide-co-glycolic acid.

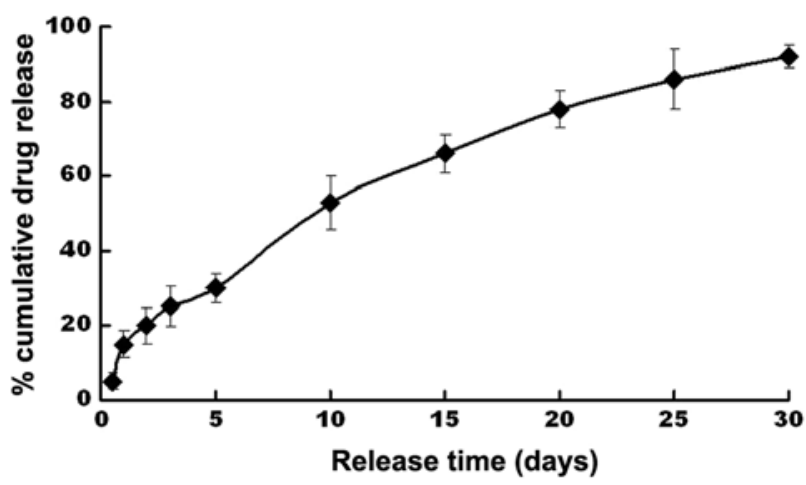

Figure 2. In vitro release profile of the 5-FU microsphere delivery system $(\mathrm{n}=5)$. 5-FU, 5-fluorouracil.

\section{Results and Discussion}

Size and surface morphology of the microsphere delivery system. Size and surface morphology of the microsphere delivery system showed smooth surfaces, spherical shape, and sizes range from 100 to $150 \mu \mathrm{m}$ (Fig. 1).

5-FU in vitro release profile and encapsulation efficiency. The in vitro release 5-FU drug profile of the microsphere delivery system in the PBS release medium is shown in Fig. 2. The in vitro drug released from the microspheres using the $\mathrm{S} / \mathrm{O} / \mathrm{hO}$ method was gradual and steady which lasted for one month. The cumulative sustained-release of 5-FU amounted to $92 \pm 4.5 \%$ of the total loading in the microspheres. The burst release amount of 5 -FU was $11 \pm 2.6 \%$ within the first day. The 5 -FU encapsulation efficiency of the microspheres using the novel method was $89.85 \pm 3.63 \%$. Considering the loss of the microsphere delivery system in the preparation process, the real encapsulation efficiency would have been much higher.

In vivo release investigation. The release of the 5-FU microsphere delivery system showed that the profile of the drug concentration in the rat plasma changed with time (Fig. 3). Mild burst release was observed from the microsphere delivery system at the initial stage of release, and the drug concentra-
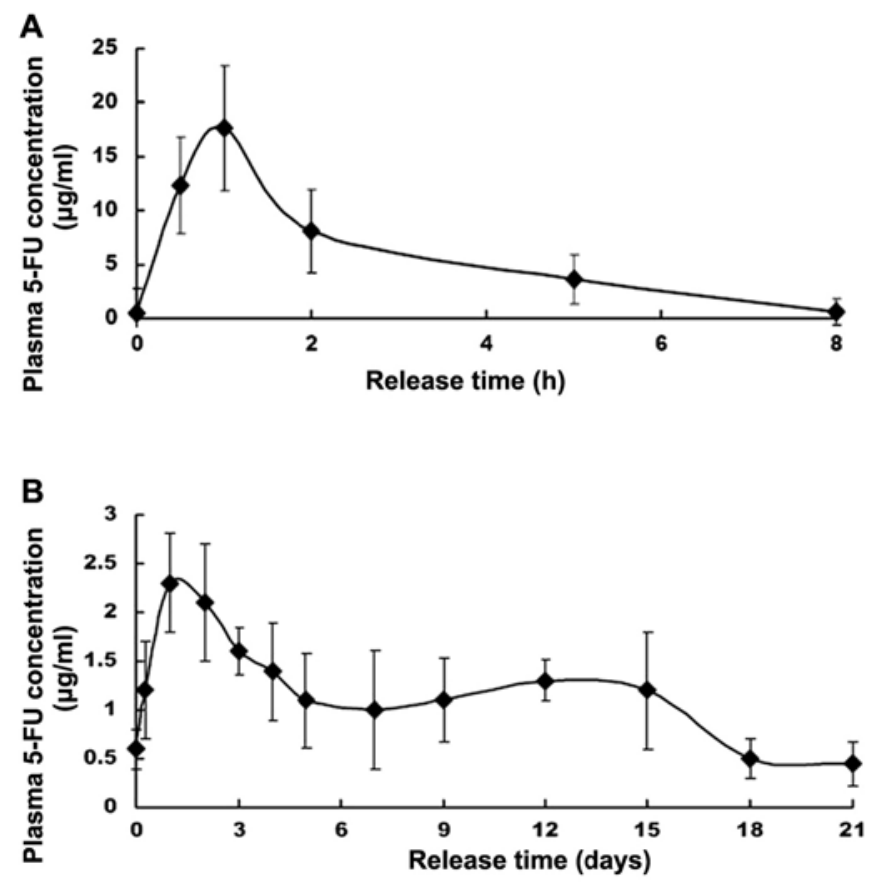

Figure 3. In vivo drug concentrations in the plasma of rats for the 5-FU microsphere delivery system (administration dose, $35 \mathrm{mg} / \mathrm{kg}$ once; $\mathrm{n}=5 \mathrm{rats}$ ). (A) 5-FU drug water solution; (B) 5-FU microsphere delivery system. 5-FU, 5-fluorouracil.

tion reached a maximum of $2.4 \pm 0.22 \mu \mathrm{g} / \mathrm{ml}$ (Fig. 3A) from the microsphere delivery system at $24 \mathrm{~h}$ after injection and then more stable concentrations were maintained from day 2 to day 20 compared with the 5-FU solution subcutaneous injection. A decreased release speed of the 5-FU drug concentration in rat plasma was determined from the straight line slope from the time of free 5-FU up to the time of maximum 5-FU drug concentration, and the circulating blood volumes for rats. Circulating blood volume in the rat was $58-70 \mathrm{ml} / \mathrm{kg}$ in terms of body weight (42), and $64 \mathrm{ml} / \mathrm{kg}$ was the suggested average value. Therefore, the speed of 5-FU drug concentration in rat plasma was $2.41 \pm 0.28 \mu \mathrm{g} / \mathrm{day}$. When the 5 -FU solution at $35 \mathrm{mg} / \mathrm{kg}$ was administered through subcutaneous injection, the maximum 5 -FU concentration was $18.11 \pm 1.02 \mu \mathrm{g} / \mathrm{ml}$, half 

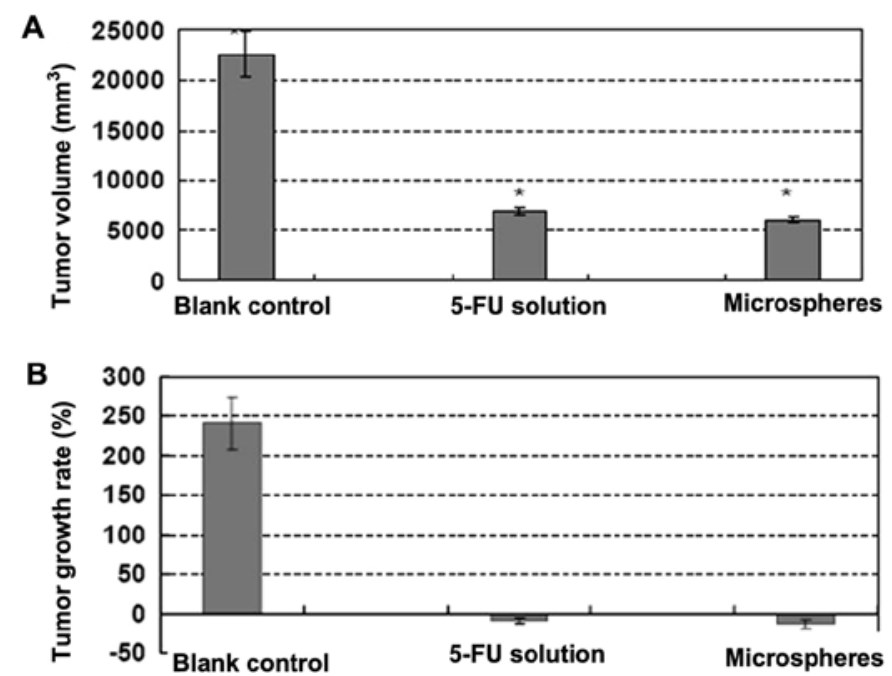

Figure 4. Antitumor effects of the blank control, 5-FU solution and microspheres $(\mathrm{P}<0.05)$ as determined by one-way ANOVA test. (A) Effects on tumor volume; (B) effects on tumor growth rate. Error bars shown the standard deviation of 6 rabbits/group. 5-FU, 5-fluorouracil.
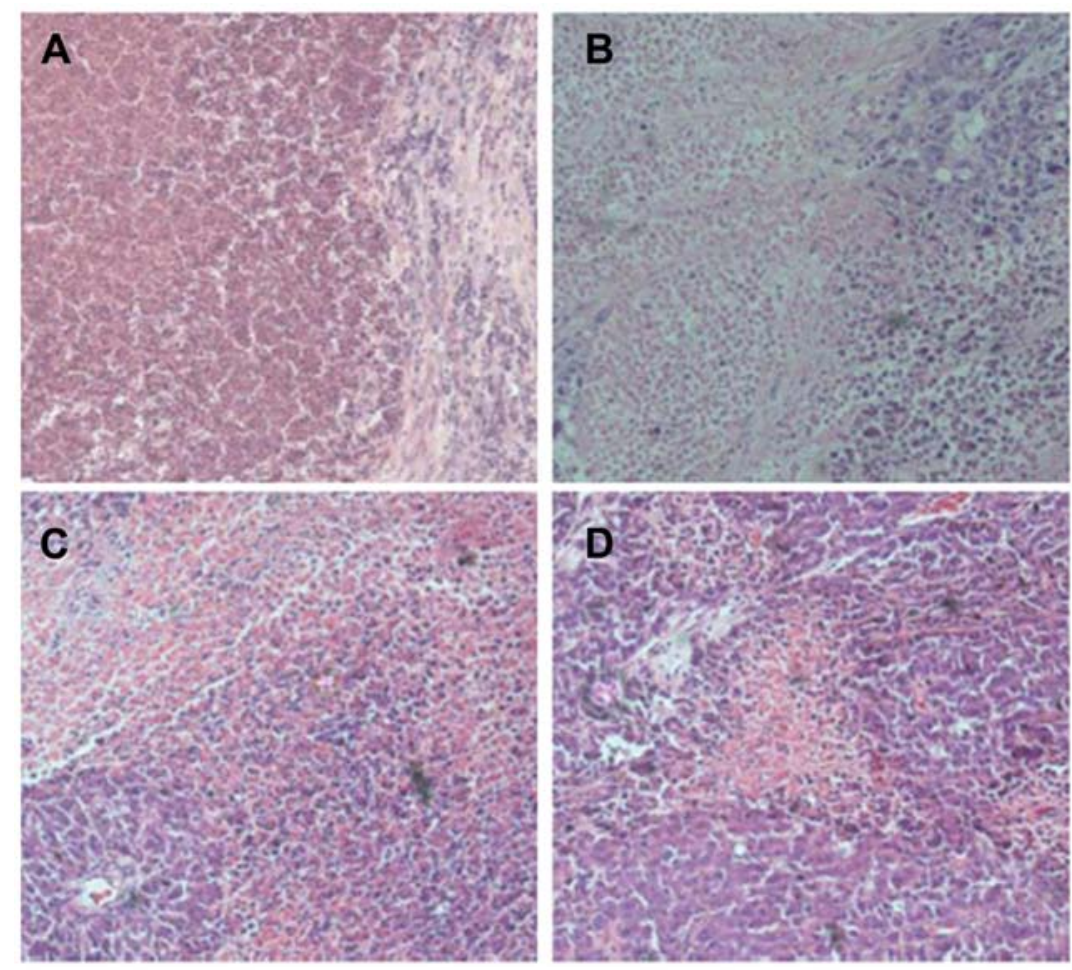

Figure 5. Hematoxylin and eosin staining of cancer tissues in rabbits following the different treatments. (A) Normal tissue, (B) following therapy with 5-FU water solution, (C) following treatment with 5-FU sustained-release microspheres and (D) tumor tissues (blank microsphere group). 5-FU, 5-fluorouracil.

an hour after the subcutaneous injection with 5-FU being determined for $5 \mathrm{~h}$ (Fig. 3B). The AUC of 5-FU injected by the 5-FU microsphere delivery system was 12 times more than that of the subcutaneous injected drug in solution. This was possibly due to the fact that the microsphere delivery system released 5-FU drug in a sustained profile, which can prolong the time of drug concentration in blood and decrease the clearance speed of 5-FU.

In vivo efficacy investigation and toxicity. In order to evaluate the therapeutic efficacy of the microsphere delivery system and 5-FU solution injection, rabbits with colon cancer were intra-tumorally administered with the 5-FU sustained-release microspheres, or intra-tumororally injected with the 5-FU solution, $0.5 \%$ sodium chloride $(30 \mathrm{mg} / \mathrm{kg} 5$-FU drug continuous infusion once as control group). Changes in tumor volume and the tumor growth inhibition rate in the rabbits were determined for 10 days. As shown in Fig. 4 the microsphere delivery system and the drug solution inhibited the tumor growth rate and achieved a significant increase in the antitumor efficacy of rabbits compared to that of the blank group. Yet, the inhibition ratio of tumor volume and tumor growth rate for the microsphere delivery system was better than that of the solution group. The toxicity related to the microsphere delivery system 
was determined by measurement of changes in body weight. Changes in the rabbit body weight in the 5-FU solution and 5-FU sustained-release microsphere groups were monitored every two days throughout this study. However, approximately similar results were found in regards to the changes in body weight between the treated groups using 5-FU solution and 5-FU sustained-release (27) microspheres ( $\mathrm{P}>0.05)$.

The intra-tumor administration of the drug delivery system to mice $(43,44)$ had an enhanced antitumor effect. The antitumor efficacy of the microsphere delivery system was also studied in rats which indicated much more significantly reduction in the mortality rate than the group administered with the water solution (45).

Pathology of the microsphere delivery system. To study the pathological effect of the microsphere delivery system, tumor samples from rabbits on day 10 were embedded, fixed, sectioned and analyzed using hematoxylin and eosin (H\&E) staining. As shown in Fig. 5 tumor tissues that received the 5-FU microspheres (Fig. 5C) exhibited certain significant necrotic areas, and had poorly defined borders and weak staining, while tumors tissues in the blank group (Fig. 5D) showed whole regions of proliferating tumor cells with a regular array and strong staining. At the same time, less necrosis was also shown in the 5-FU solution group (Fig. 5B).

In conclusion, in the present study, the 5-FU sustainedrelease microspheres using a novel $\mathrm{S} / \mathrm{O} / \mathrm{hO}$ method was developed for the 5-FU sustained-release, and local targeting in tumor tissues. 5-FU sustained-release microspheres were fabricated with biocompatible and biodegradable PLGA. According to our test data, the 5-FU drug sustained-release effect from the 5-FU sustained-release microspheres in vitro and in vivo was for $\sim 30$ and 21 days.

In addition, the antitumor effects were also studied in rabbits, which demonstrated an enhanced antitumor effect by administering 5-FU sustained-release microsphere delivery system compared to the intra-tumoral administration of the 5-FU water solution. Local administration of the 5-FU microsphere delivery system enhanced the drug concentration in the cancer tissues. Therefore, the microsphere delivery system may improve the antitumor effects and reduce the 5-FU toxicity in normal tissues. As a result, intra-tumoral administration of the microsphere delivery system may provide better local treatment for cancer chemotherapy against solid tumors.

\section{Acknowledgements}

The present study was supported by Shanghai's Science and Technology Achievements Transformation and Industrialization (no. 13401900801), the National Science Foundation of China (no. 81001416, 81001407), and Funds for Interdisciplinary Projects of Medicine and Engineering by Shanghai JiaoTong University (nos. YG2013MS52 and YG2013MS62).

\section{References}

1. Moehler M, Teufel A and Galle PR: New chemotherapeutic strategies in colorectal cancer. Recent Results Cancer Res 165: 250-259, 2005

2. Segura C, Afchain P, de Gramont A, André T; Gercor (French Oncology Research Group): Adjuvant treatment of colorectal cancer. Bull Cancer 93: 683-690, 2006 (In French).
3. El-Khoueiry $A B$ and Lenz HJ: Should continuous infusion 5 -fluorouracil become the standard of care in the USA as it is in Europe? Cancer Invest 24: 50-55, 2006.

4. Goldberg RM, Sargent DJ, Morton RF, et al: Randomized controlled trial of reduced-dose bolus fluorouracil plus leucovorin and irinotecan or infused fluorouracil plus leucovorin and oxaliplatin in patients with previously untreated metastatic colorectal cancer: a North American Intergroup Trial. J Clin Oncol 24: 3347-3353, 2006.

5. Günzburg WH, Löhr M and Salmons B: Novel treatments and therapies in development for pancreatic cancer. Expert Opin Investig Drugs 11: 769-786, 2002.

6. Li D, Xie K, Wolff R and Abbruzzese JL: Pancreatic cancer. Lancet 363: 1049-1057, 2004.

7. Jorizzo J: Topical treatment of actinic keratosis with fluorouracil: is irritation associated with efficacy? J Drugs Dermatol 3: 21-26, 2004.

8. Gupta AK, Davey V and Mcphail H: Evaluation of the effectiveness of imiquimod and 5-fluorouracil for the treatment of actinic keratosis: critical review and meta-analysis of efficacy studies. J Cutan Med Surg 9: 209-214, 2005.

9. Yarde Y, Baselga J and Miles D: Molecular approach to breast cancer treatment. Semin Oncol 31 (Suppl 10): S6-S13, 2004.

10. Hutchins LF, Green SJ, Ravdin PM, et al: Randomized, controlled trial of cyclophosphamide, methotrexate, and fluorouracil versus cyclophosphamide, doxorubicin, and fluorouracil with and without tamoxifen for high-risk, node-negative breast cancer: treatment results of Intergroup Protocol INT-0102. J Clin Oncol 23: 8313-8321, 2005.

11. Eniu A, Carlson RW, Aziz Z, et al: Breast cancer in limitedresource countries: treatment and allocation of resources. Breast J 12 (Suppl 1): S38-S53, 2006.

12. Roüessé J, de la Lande B, Bertheault-Cvitkovic F, et al: A phase III randomized trial comparing adjuvant concomitant chemoradiotherapy versus standard adjuvant chemotherapy followed by radiotherapy in operable node-positive breast cancer: final results. Int J Radiat Oncol Biol Phys 64: 1072-1080, 2006.

13. Roullin VG, Mege M, Lemaire L, et al: Influence of 5-fluorouracil-loaded microsphere formulation on efficient rat glioma radiosensitization. Pharm Res 21: 1558-1563, 2004.

14. Larina IV, Evers BM, Ashitkov TV, et al: Enhancement of drug delivery in tumors by using interaction of nanoparticles with ultrasound radiation. Technol Cancer Res Treat 4: 217-226, 2005.

15. Muvaffak A, Gurhan I, Gunduz U and Hasirci N: Preparation and characterization of a biodegradable drug targeting system for anticancer drug delivery: microsphere-antibody conjugate. J Drug Target 13: 151-159, 2005.

16. Santhi K, Dhanaraj SA, Joseph V, et al: A study on the preparation and anti-tumor efficacy of bovine serum albumin nanospheres containing 5-fluorouracil. Drug Dev Ind Pharm 28: 1171-1179, 2002.

17. Fournier E, Passirani C, Vonarbourg A, et al: Therapeutic efficacy of novel 5-FU-loaded PMM 2.1.2-based microspheres on C6 glioma. Int J Pharm 268: 31-35, 2003.

18. Menei P, Jadaud E, Faisant N, et al: Stereotaxic implantation of 5-fluorouracil-releasing microspheres in malignant glioma. Cancer 100: 405-410, 2004.

19. Blanco MD, Garcia O, Gomez C, et al: In-vivo drug delivery of 5-fluorouracil using poly(2-hydroxyethyl methacrylate-coacrylamide) hydrogels. J Pharm Pharmacol 52: 1319-1325, 2000.

20. Zambito Y, Baggiani A, Carelli V, et al: Matrices for site-specific controlled-delivery of 5-fluorouracil to descending colon. J Control Release 102: 669-677, 2005.

21. Simeonova M, Velichkova R, Ivanova G, et al: Poly(butylcyanoacrylate) nanoparticles for topical delivery of 5-fluorouracil. Int J Pharm 263: 133-140, 2003.

22. Nair KL, Jagadeeshan S, Nair SA and Kumar GS: Biological evaluation of 5-fluorouracil nanoparticles for cancer chemotherapy and its dependence on the carrier, PLGA. Int J Nanomed 6: 1685-1697, 2011.

23. Ma J, Chen CS, Blute T and Waxman DJ: Antiangiogenesis enhances intratumoral drug retention. Cancer Res 71: 2675-2685, 2011.

24. Banerjee S, Sen K, Pal TK and Guha SK: Poly(styrene-co-maleic acid)-based $\mathrm{pH}$-sensitive liposomes mediate cytosolic delivery of drugs for enhanced cancer chemotherapy. Int J Pharm 436: 786-797, 2012.

25. Ragab DM, Rohani S and Consta S: Controlled release of 5-fluorouracil and progesterone from magnetic nanoaggregates. Int $\mathrm{J}$ Nanomed 7: 3167-3189, 2012. 
26. Du JZ, Du XJ, Mao CQ and Wang J: Tailor-made dual $\mathrm{pH}$-sensitive polymer-doxorubicin nanoparticles for efficient anticancer drug delivery. J Am Chem Soc 133: 17560-17563, 2011.

27. Ren X, Zheng N, Gao Y, et al: Biodegradable three-dimension micro-device delivering 5 -fluorouracil in tumor bearing mice. Drug Deliv 19: 36-44, 2012.

28. Choi KY, Yoon HY, Kim JH, et al: Smart nanocarrier based on PEGylated hyaluronic acid for cancer therapy. ACS Nano 5: 8591-8599, 2011.

29. Li J, Hou N, Faried A, et al: Inhibition of autophagy augments 5 -fluorouracil chemotherapy in human colon cancer in vitro and in vivo model. Eur J Cancer 46: 1900-1909, 2010.

30. Arias JL: Novel strategies to improve the anticancer action of 5-fluorouracil by using drug delivery systems. Molecules 13: 2340-2369, 2008.

31. Zhu Y, Ikoma T, Hanagata $\mathrm{N}$ and Kaskel S: Rattle-type $\mathrm{Fe}_{3} \mathrm{O}_{4} @$ $\mathrm{SiO}_{2}$ hollow mesoporous spheres as carriers for drug delivery. Small 6: 471-478, 2010.

32. Xu D, Hu Z, Su J, et al: Micro and nanotechnology for intracellular delivery therapy protein. Nano-Micro Letters 4: 118-123, 2012.

33. Yuan W and Liu Z: Surgical wound healing using hemostatic gauze scaffold loaded with nanoparticles containing sustainedrelease granulocyte colony-stimulating factor. Int J Nanomedicine 6: 3139-3149, 2011

34. Hong X, Wei L, Ma L, Chen Y, et al: Novel preparation method for sustained-release PLGA microspheres using water-in-oil-inhydrophilic-oil-in-water emulsion. Int J Nanomed 8: 2433-2441, 2013.

35. Yuan W, Wu F, Guo M and Jin T: Development of protein delivery microsphere system by a novel S/O/O/W multi-emulsion. Eur J Pharm Sci 36: 212-218, 2009

36. Liu G, Hong X, Jiang M and Yuan W: Sustained-release G-CSF microspheres using a novel solid-in-oil-in-oil-in-water emulsion method. Int J Nanomed 7: 4559-4569, 2012.
37. Yuan W and Liu Z: Controlled-release and preserved bioactivity of proteins from (self-assembled) core-shell double-walled microspheres. Int J Nanomed 7: 257-270, 2012

38. Yuan W, Zhang Y, Wu F, et al: Preparation of protein-loaded sustained-release microspheres via 'solid-in-oil-in-hydrophilic oil-in-ethanol (S/O/hO/E)' emulsification. Colloids Surf B Biointerfaces 79: 326-333, 2010.

39. Zhao H, Wu F, Cai Y, Chen Y, et al: Local antitumor effects of intratumoral delivery of rlL-2 loaded sustained-release dextran/ PLGA-PLA core/shell microspheres. Int J Pharm 450: 235-240, 2013.

40. Xu L, Wu F, Yuan W and Jin T: Controlled-release implant system formulated using biodegradable hemostatic gauze as scaffold. Int J Pharm 355: 249-258, 2008.

41. Buckpitt AR and Boyd MR: A sensitive method for determination of 5-fluorouracil and 5-fluoro-2'-deoxyuridine in human plasma by high-pressure liquid chromatography. Anal Biochem 106: 432-437, 1980.

42. Diehl KH, Hull R, Morton D, et al: A good practice guide to the administration of substances and removal of blood, including routes and volumes. J Appl Toxicol 21: 15-23, 2001.

43. Konishi M, Tabata Y, Kariya M, et al: In vivo anti-tumor effect of dual release of cisplatin and adriamycin from biodegradable gelatin hydrogel. J Control Release 103: 7-19, 2005.

44. Obara K, Ishihara M, Ozeki Y, et al: Controlled release of paclitaxel from photocrosslinked chitosan hydrogels and its subsequent effect on subcutaneous tumor growth in mice. J Control Release 110: 79-89, 2005.

45. Menei P, Boisdron-Celle M, Crousé A, et al: Effect of stereotactic implantation of biodegradable 5-fluorouracil-loaded microspheres in healthy and C6 glioma-bearing rats. Neurosurgery 39: 117-124, 1996. 